\title{
Shower universality reconstruction of data from the Pierre Auger Observatory and validations with hadronic interaction models
}

\author{
Ariel Bridgeman $^{a}$ for the Pierre Auger Collaboration ${ }^{b}$ \\ ${ }^{a}$ Institute for Nuclear Physics, Karlsruhe Institute of Technology, Karlsruhe, Germany \\ ${ }^{b}$ Observatorio Pierre Auger, Av. San Martín Norte 304, 5613 Malargüe, Argentina \\ E-mail: auger_spokespersons@fnal.gov \\ Full author list: http://www.auger.org/archive/authors_icrc_2017.html
}

\begin{abstract}
The determination of the mass composition of ultrahigh-energy cosmic rays remains one of the biggest challenges of astroparticle physics. We will show that the paradigm of shower universality can be applied to accurately reconstruct the properties of air showers, which includes information about the primary mass. The reconstruction is based solely on data from the surface detector of the Pierre Auger Observatory, which operates with a duty cycle of nearly $100 \%$. In contrast to purely empirical reconstruction methods, the foundation of the universality approach lies in physics models of the signal and arrival time distributions of secondary particles in air showers. In this contribution, results of the universality reconstruction are compared to their counterparts from the measurements of the Auger fluorescence detector. The focus of these comparisons is on the depth of the shower maximum, primary energy, and geometry. In addition, we extend these eventby-event comparisons to air shower simulations of various hadronic interaction models, primary masses, and energies. We will also present the performance of the method in estimating the muon content of air showers by comparing the true and reconstructed muon number for simulated showers.
\end{abstract}

35th International Cosmic Ray Conference - ICRC2017

10-20 July, 2017

Bexco, Busan, Korea 


\section{Introduction}

Determining the mass composition of ultrahigh-energy cosmic rays (UHECRs) at the highest energies remains one of the greatest challenges of experimental astroparticle physics. Together with the primary energy, knowledge of the primary mass is of critical importance to distinguish between different astrophysical scenarios and to find the sources of UHECRs. Via its accurate measurement of the depth of the shower maximum, the fluorescence detector (FD) of the Pierre Auger Observatory [1] has provided valuable insight into the mass composition of UHECRs [2]. However, the duty cycle of the FD is limited to around $13 \%$, as measurements are only possible on clear, moonless nights. While there is a wealth of data acquired with the extensive surface detector (SD) of Auger [3, 4] over the last 12 years, air showers of primaries with different masses create nearly indistinguishable signals in the water-Cherenkov detectors (WCDs).

In order to exploit the data from the SD for mass identification, a phenomenological methodknown as shower universality - is used, which is based on the underlying physics of an extensive air shower. The average properties of the cascade of secondary particles depend mostly on the primary energy and the stage of shower development. As the shower-to-shower fluctuations are minimal compared to the overall shower development, a universal description of four uniquely-defined particle components may be employed. Using signal (Section 2.1) and time (Section 2.2) models, the universality approach allows us to reliably estimate the time-dependent signals of the different particle components in triggered surface detectors. This enables the reconstruction of macroscopic parameters - like the energy, the depth of the shower maximum, and the muonic content of an extensive air shower (Section 3). Comparisons between those results and measurements with the FD are discussed in this paper in Section 4.

\section{Air shower universality}

Our air shower models are based on the universal description of the time-dependent signals of four distinct groups of secondary particles: 1 . muons $(\mu)$, 2. electromagnetic particles ${ }^{1}$ from high-energy $\pi^{0}$ decays $(e \gamma)$, 3. electromagnetic particles from muon decay or interactions $(e \gamma(\mu))$, and 4. electromagnetic particles from the decay of low-energy hadrons $(e \gamma(\mathrm{had}))$. The underlying universal behavior of the shower development has been previously studied ([5] and references therein). In particular, the development of the electromagnetic shower has been thoroughly explored [5-9]. To include showers of hadrons with $A>1$ in the description, an additional parameter is needed to take into account the muon content and its development - the relative number of muons $R_{\mu}{ }^{2}$ [10-12]. Each particle component except the main electromagnetic cascade is correlated to the overall muon scale. The stage of shower development is captured with $\Delta X$-the integrated atmospheric overburden between the shower maximum $X_{\max }$ and the projected position of the station in the shower plane. As depicted in Fig. 1a, $\Delta X$ differs for stations at the same radial yet differing azimuthal location.

\footnotetext{
${ }^{1}$ While all of the listed particles are electromagnetic, we use this nomenclature to refer only to electrons, positrons, and photons. This particular component corresponds with the main electromagnetic cascade.

${ }^{2}$ See Section 2.1 for more information.
} 


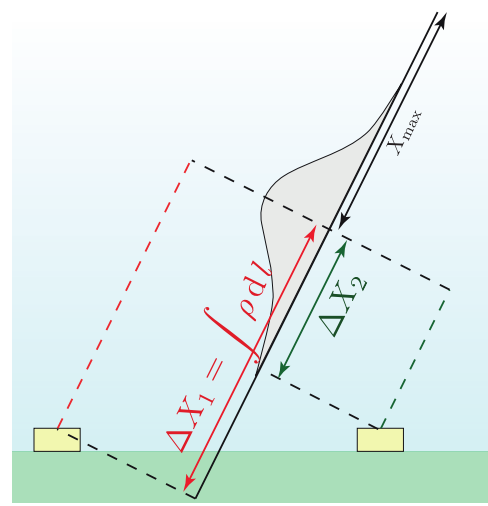

(a)

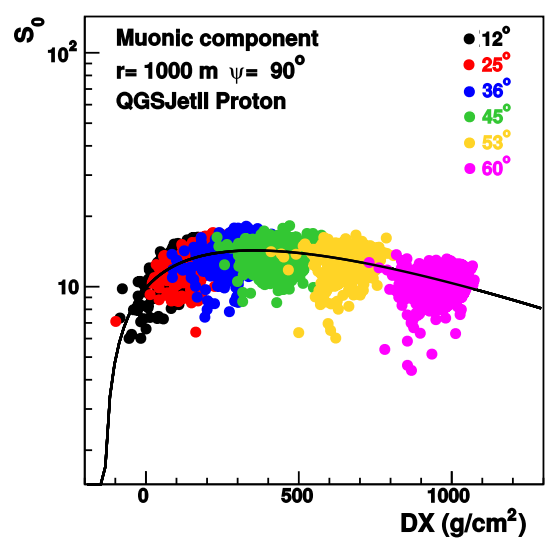

(b)

Figure 1: (a) The longitudinal development $\Delta X$ is the distance between a station and the $X_{\max }$. Stations at $\psi=0$ and $\psi=180^{\circ}$ illustrate the azimuthal dependence of the observed shower stage [12]. (b) Comparison of simulations with various zenith angles to the parameterization (solid line) [11] for the $\Delta X$ dependence of the measured signal $S_{0}$ in a WCD expected for the muonic component signal [13].

To derive the parameterizations for the signal and time models, a CORSIKA [14] shower library - consisting of proton and iron primaries simulated with QGSJET-II.03 and EPOS-1.99— was utilized [13]. Both models are derived from SD simulations which incorporate the hardware and calibration properties of the SD. As shown in this paper, the shower universality approach was extensively validated with new air shower simulations, i.e. QGSJET-II.04 [15] and EPOS-LHC [16], and successfully applied to hybrid data.

\subsection{Signal model}

For each particle component, the signal $S$ in a WCD is calculated starting with the signal $S_{0}$ observed in an ideal $10 \mathrm{~m}^{2}$ spherical detector. Afterwards, the asymmetries due to the detector geometry and particle production are parameterized. Employing both the parameterization of asymmetries and the ideal signal $S_{0}$, the expected signal of a real detector is described as [13],

$$
S_{0}(\Delta X, E)=S_{\max }\left(\frac{E}{10^{19} \mathrm{eV}}\right)^{\gamma}\left(\frac{\Delta X-\Delta X_{0}}{\Delta X_{\max }-\Delta X_{0}}\right)^{\frac{\Delta X_{\max }-\Delta X_{0}}{\lambda(E)}} \exp \left(\frac{\Delta X_{\max }-\Delta X}{\lambda(E)}\right),
$$

with $\lambda(E)=\lambda_{0}+f_{\lambda} \lg \left(E / 10^{19} \mathrm{eV}\right)$. For muons and muon decay products, the energy evolution $f_{\lambda}$ vanishes. Results of the longitudinal parameterization $S_{0}(\Delta X)$ for the muonic component is shown in Fig. 1b.

For this comparison, signals from different primary energies are included, corrected for via $\lambda(E)$, and evaluated at a radial distance of $1000 \mathrm{~m}$. The parameters of the longitudinal description $-S_{\max }, \Delta X_{\max }, \gamma, \lambda_{0}$, and $f_{\lambda}$-depend on the distance to the shower core, and subsequent parameterizations for these variables were found. In particular, $S_{\max }(r)$ is best described with a power-law lateral distribution function (LDF), which is independent of the mass composition and hadronic interaction model at $10^{19} \mathrm{eV}$. When coupled together, the parameterizations of the longitudinal and the lateral distributions of the ideal signal establish the model $S_{0}(\Delta X, r, E)$. The signal $S$ in a real detector takes into account truncation asymmetries due to the presence of the ground and a 


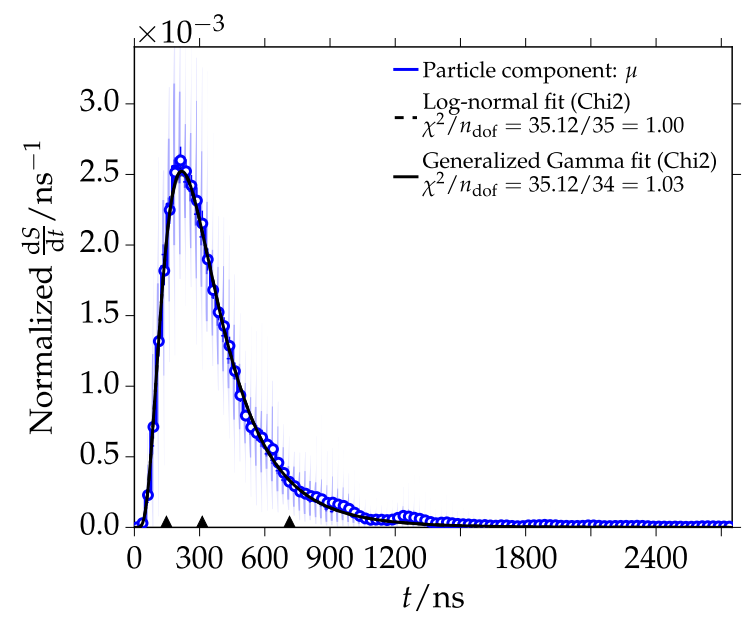

(a)

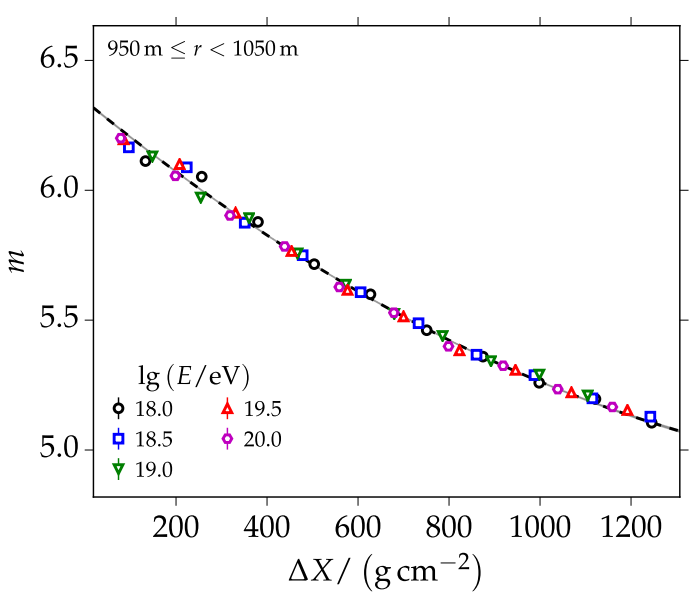

(b)

Figure 2: (a) Average time distributions of ground signals in air showers initiated by proton, carbon, and iron primaries with an energy of $10^{19} \mathrm{eV}$ and a zenith angle of $0^{\circ}$. Shown are the average traces for specific distances to $X_{\max }$ for the muonic particle component. The traces are normalized such that their integral is equal to one. (b) Model description of the $\Delta X$ dependence of the mean parameter $m$ from Eq. (2.2) for muons [12].

non-spherical detector. For almost all regions of the parameter space, deviations in the description of $S$ are smaller than $5 \%$ [13]. To derive $R_{\mu}$, the signal model is used with the reference signal $S_{0, \mu}^{\text {ref }}$ from a QGSJET-II.03 proton shower with an energy of $10^{19} \mathrm{eV}$ and local shower azimuth of $\psi=90^{\circ}$.

\subsection{Time model}

The time-dependent signal in a WCD depends on the arrival time distribution of secondary particles and the detector response. Due to the various particle types and energies, an analytical expression of the detector response is hard to derive, and expensive detector simulations are necessary. To speed-up those simulations, the detector response was tabulated [11]. Then, independently for each particle component, we parametrize the arrival time distributions after the simulation of the detector response with $\overline{\mathrm{Off}} \underline{\text { line }}$ [17]. The procedure described here is an extension of what is described in [18].

First, traces are divided into bins with respect to distance to the core $r$, distance to shower maximum $\Delta X$, primary energy $E$, zenith angle $\theta$, and azimuth angle $\psi$. Within each $(r, \psi, \theta, E, \Delta X)$ bin, a weighted average of all traces is calculated, and fit with a chi-squared minimization. An example for the muonic particle component is depicted in Fig. 2a. The best fits were achieved with a log-normal or a generalized gamma distribution ${ }^{3}$.

Using the log-normal distribution, the evolution of the shape parameters mean $m$ and width $s$ is described with

$$
f_{\mathrm{m}, \mathrm{s}}(\Delta X, \psi, \theta, E)=f_{\Delta X}(\Delta X)+f_{\mathrm{geo}}(\theta, \psi, \Delta X)+f_{\lg E}(\lg E, \Delta X)
$$

\footnotetext{
${ }^{3}$ The generalized gamma distribution has three parameters, denoted as $m, s$, and $\ell$. For $\ell=0$, it reduces to a $\log$-normal distribution with $m$ and $s$ - which are proportional to the mean and standard deviation.
} 


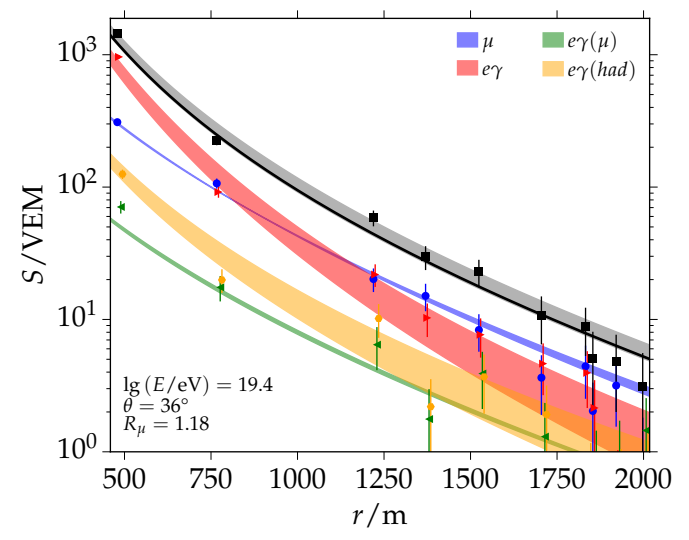

(a)

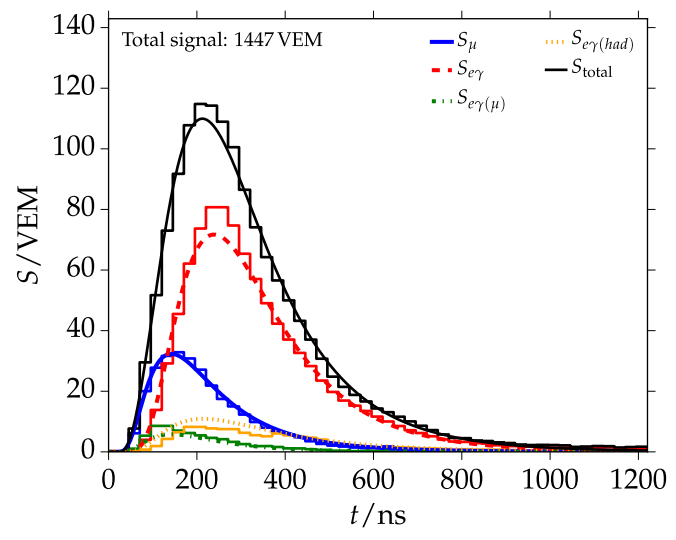

(b)

Figure 3: (a) Example LDFs in the universality reconstruction of a simulated event. The sum of component predictions is fit to the total signals (black). A comparison of the LDF components and their model predictions are given. (b) Results of the time fit of the hottest station. For comparison, the component traces (histograms) are plotted against the model predictions (smooth lines), which come from the fit of the total trace (black) [12].

where

$$
\begin{aligned}
f_{\Delta X}(\Delta X) & =a_{\Delta X}+\Delta X_{\text {ref }}\left(b_{\Delta X}+\Delta X_{\text {ref }}\left(c_{\Delta X}+d_{\Delta X} \Delta X_{\text {ref }}\right)\right), \\
f_{\text {geo }}(\theta, \psi, \Delta X) & =\sin \theta\left(a_{\text {geo }} \cos \psi+b_{\text {geo }} \Delta X_{\text {ref }}\right), \\
f_{E}(\lg E, \Delta X) & =\lg E_{\text {ref }}\left(a_{\lg E}+b_{\lg E} \Delta X_{\text {ref }}\right), \\
\Delta X_{\text {ref }} & =\Delta X /\left(750 \mathrm{~g} \mathrm{~cm}^{-2}\right), \quad \text { and } \\
\lg E_{\text {ref }} & =\lg (E / \mathrm{eV})-19 .
\end{aligned}
$$

Depending on the particle component and parameter, the number of fit parameters is reduced. The model holds for a specific core distance range with width $\Delta r$. To fully capture dependencies on the core distance, Eq. (2.2) is first fit in each of the available core distance bins, and then analytical expressions as a function of $r$ are found. Results on the $\Delta X$ dependence of $m$ for the muonic particle component is shown in Fig. 2b. The accuracy of the description of $m$ is better than $1 \%$ for all particle components and dependencies. As there are more fluctuations in the spread $s$, deviations up to $5 \%$ occur for the muonic and the electromagnetic components. For the remaining particle components, most of the deviations are within $5 \%$.

\section{Reconstruction algorithm}

The universality reconstruction employs the signal and time models to fit the measured particle traces and provide estimates of air shower properties. The reconstruction is composed of several aspects: a simultaneous fit of the distribution of time traces, the start times of triggered stations, and the lateral distribution of the total measured signals. A fit of time distributions is only attempted for stations with large enough signals (at least five time bins with a signal exceeding 0.7 VEM). The universality reconstruction is comprised of 9 parameters: the core position $\vec{x}$, the relative core time $t_{c}$, the shower arrival direction $(\theta, \phi)$, energy $E$, shower maximum $X_{\max }$, and relative muon number $R_{\mu}$. Depending on the reconstruction method employed, these parameters may be fixed or 

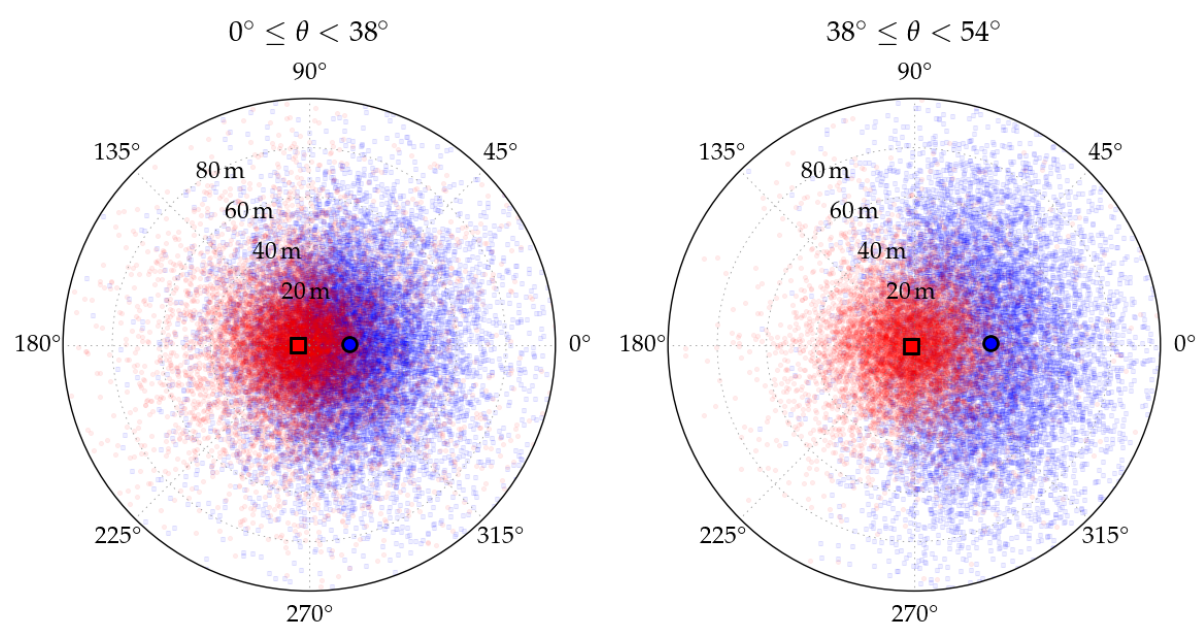

Figure 4: The universality reconstruction yields an—on average—unbiased core position (red) with respect to the MC core, whereas the core position of the SD reconstruction (blue) is systematically biased.

free during the minimization of the total log-likelihood. In Fig. 3, the lateral signal distributions and time distributions for each particle component resulting from the universality reconstruction of a proton event with $\lg (E / \mathrm{eV})=19.4$ are depicted together with details about the simulated event. As universality utilizes the SD-whose energy is calibrated with the FD-the universality reconstruction fixes the energy to that found via the SD reconstruction [1] and then simultaneously fits the remaining 8 parameters.

\section{Performance in simulations and with hybrid data}

For the validation of the universality reconstruction, we study biases and resolutions of the reconstructed quantities. For this purpose, we used a CORSIKA shower library with more than 60000 proton and iron simulations of QGSJET-II.04 and EPOS-LHC. Due to its explicit dependence on $\Delta X$, the universality reconstruction naturally accounts for the asymmetric azimuthal dependence of the signal. As a consequence, as seen in Fig. 4, the universality reconstructed core is unbiased relative to the MC core. In Fig. 5a and Fig. 5b, an unbiased estimate of $X_{\max }$ and $R_{\mu}$ is observed for QGSJET-II.04 proton and iron simulations. Hybrid data also exhibits an unbiased reconstruction. Due to small differences between the arrival time distributions of particles in QGSJET-II.04 and EPOS-LHC simulations, a bias is seen for EPOS-LHC simulations in Fig. 5a. The resolution of $X_{\max }$ ranges from 80 to $30 \mathrm{~g} \mathrm{~cm}^{-2}$ at the highest energies. Due to their larger muon content, iron simulations are reconstructed with a smaller resolution, on average. The resolution of $R_{\mu}$ approaches $10 \%$ at the highest energies. The correlation between $R_{\mu}$ and $X_{\max }$ is depicted in Fig. 5c and Fig. $5 \mathrm{~d}$. The bias in the reconstructed $X_{\max }$ of EPOS-LHC causes slight differences in the shape of the correlations.

\section{Conclusions}

Shower universality is based on the intrinsic physics properties of extensive air showers. Air 

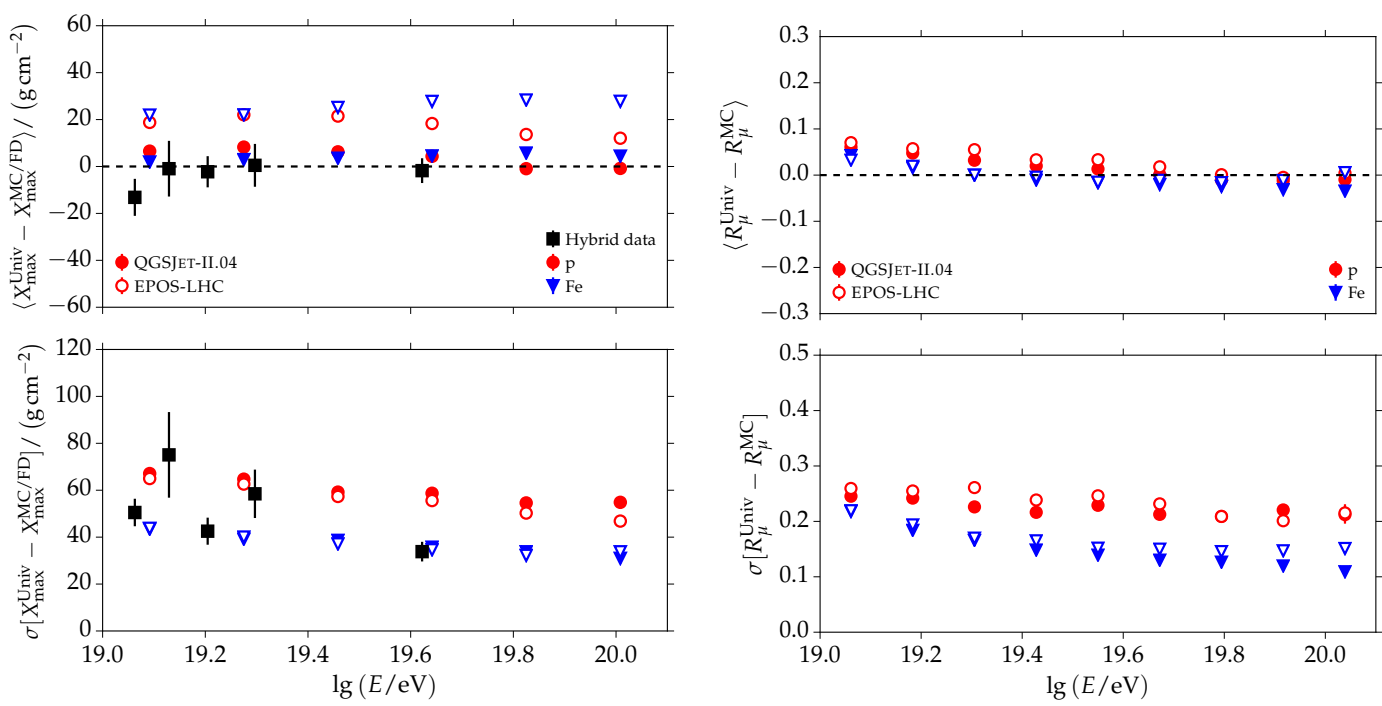

(a)

(b)

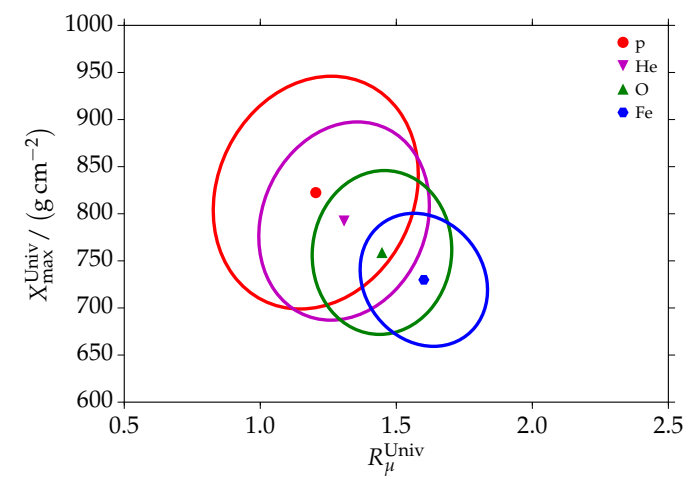

(c)

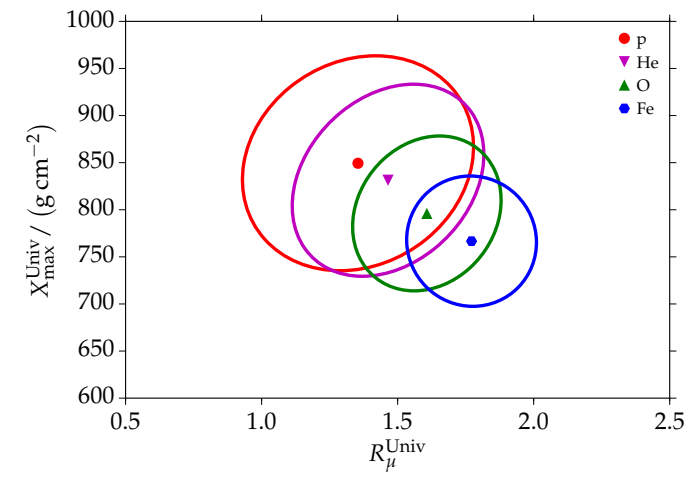

(d)

Figure 5: The bias and resolution plots for (a) $X_{\max }$ and (b) $R_{\mu}$ for different primaries and hadronic interaction models. A comparison between the FD and universality reconstructed $X_{\max }$ is also given. The correlation between $R_{\mu}$ and $X_{\max }$ for different primaries of (c) QGSJET-II.04 and (d) Epos-LHC. Shown are the $1 \sigma$ contours of reconstructed quantities.

shower simulations with contemporary interaction models were used to extract models of the timedependent signals in surface detectors for different particle components. We demonstrated that a reconstruction algorithm based on these assumptions and only SD information performs very well for both simulations and data. In particular, a direct comparison with FD measurements highlights an unbiased estimate of $X_{\max }$. This enables the reconstruction of mass-sensitive parameters with the full SD event statistics at the highest energies and with a competitive resolution. Together with the upgraded detectors of AugerPrime, the method will give access to event-by-event estimates of the primary mass at the highest energies.

\section{References}

[1] Pierre Auger Collaboration, J. Abraham et al., Properties and performance of the prototype instrument for the Pierre Auger Observatory, Nucl. Instrum. Meth. A 523 (2004), no. 1-2 50 - 95. 
[2] Pierre Auger Collaboration, A. Aab et al., Depth of Maximum of Air-Shower Profiles at the Pierre Auger Observatory: Composition Implications, Phys. Rev. D (2014).

[3] Pierre Auger Collaboration, A. Aab et al., The Pierre Auger Cosmic Ray Observatory, Nucl. Instrum. Meth. A 798 (2015) $172-213$.

[4] Pierre Auger Collaboration, I. Allekotte et al., The Surface Detector System of the Pierre Auger Observatory, Nucl. Instrum. Meth. A 586 (2008) 409-420.

[5] P. Lipari, The Concepts of 'Age' and 'Universality' in Cosmic Ray Showers, Phys. Rev. D 79 (2008) 063001.

[6] A. M. Hillas, Angular and energy distributions of charged particles in electron photon cascades in air, J. Phys. G 8 (1982) 1461-1473.

[7] M. Giller, A. Kacperczyk, J. Malinowski, W. Tkaczyk, and G. Wieczorek, Similarity of extensive air showers with respect to the shower age, J. Phys. G 31 (2005) 947-958.

[8] P. Lipari, Universality of cosmic ray shower development, Nucl. Phys. Proc. Suppl. 196 (2009) 309-318.

[9] S. Lafebre, R. Engel, H. Falcke, J. Hörandel, T. Huege, J. Kuijpers, and R. Ulrich, Universality of electron-positron distributions in extensive air showers, Astropart. Phys. 31 (2009) 243-254.

[10] F. Schmidt, M. Ave, L. Cazon, and A. S. Chou, A Model-Independent Method of Determining Energy Scale and Muon Number in Cosmic Ray Surface Detectors, Astropart. Phys. 29 (2008) 355-365.

[11] M. Ave, R. Engel, J. Gonzalez, D. Heck, T. Pierog, and M. Roth, Extensive air shower universality of ground particle distributions, Proc. of 31st Int. Cosmic Ray Conf., Beijing (2011) \#1025.

[12] M. Roth, M. Ave, and A. Schulz, A universal description of temporal and lateral distributions of ground particles in extensive air showers, in ICRC Proceedings 2015, 2015.

[13] M. Ave, R. Engel, M. Roth, and A. Schulz, A generalized description of the signal size in extensive air shower detectors and its applications, Astropart. Phys. 87 (2017) 23 - 39.

[14] D. Heck, G. Schatz, T. Thouw, J. Knapp, and J. Capdevielle, CORSIKA: A Monte Carlo code to simulate extensive air showers, Forschungszentrum Karlsruhe Report FZKA (1998) 6019.

[15] S. Ostapchenko, Monte Carlo treatment of hadronic interactions in enhanced Pomeron scheme: QGSJET-II model, Phys. Rev. D 83 (2011), no. 1.

[16] T. Pierog and K. Werner, EPOS Model and Ultra High Energy Cosmic Rays, Nucl. Phys. B Proc. Suppl. 196 (2009) 102-105.

[17] S. Argirò, S. Barroso, J. Gonzalez, L. Nellen, T. Paul, T. Porter, L. P. Jr., M. Roth, R. Ulrich, and D. Veberič, The Offline software framework of the Pierre Auger Observatory, Nucl. Instrum. Meth. A $\mathbf{5 8 0}$ (2007) 1485-1496.

[18] M. Ave, M. Roth, and A. Schulz, A generalized description of the time dependent signals in extensive air shower detectors and its applications, Astropart. Phys. 88 (2017) 46 - 59. 